\title{
Distribution and Quantification of 1,2-Propylene Glycol Enantiomers in Baijiu
}

\author{
Hao Xu ${ }^{1}$, Yifeng Dai ${ }^{1,2, *}$, Shuyi Qiu ${ }^{1}$, Baoguo Sun ${ }^{2}$ and Xiangyong Zeng ${ }^{1}$ \\ 1 Guizhou Province Key Laboratory of Fermentation Engineering and Biopharmacy, \\ School of Liquor and Food Engineering, Guizhou University, Guiyang 550025, China; \\ xuhao20211108@163.com (H.X.); syqiu@gzu.edu.cn (S.Q.); xyzeng1@gzu.edu.cn (X.Z.) \\ 2 Beijing Advanced Innovation Center for Food Nutrition and Human Health, \\ Beijing Technology and Business University, Beijing 100048, China; sunbg@btbu.edu.cn \\ * Correspondence: yfdai3@gzu.edu.cn
}

Citation: Xu, H.; Dai, Y.; Qiu, S.; Sun, B.; Zeng, X. Distribution and Quantification of 1,2-Propylene Glycol Enantiomers in Baijiu. Foods 2021, 10, 3039. https://doi.org/ $10.3390 /$ foods10123039

Academic Editor: Vincenzo Gerbi

Received: 5 November 2021

Accepted: 3 December 2021

Published: 7 December 2021

Publisher's Note: MDPI stays neutral with regard to jurisdictional claims in published maps and institutional affiliations.

Copyright: (c) 2021 by the authors. Licensee MDPI, Basel, Switzerland. This article is an open access article distributed under the terms and conditions of the Creative Commons Attribution (CC BY) license (https:// creativecommons.org/licenses/by/ $4.0 /)$.
Abstract: Enantiomers of 1,2-Propylene glycol (1,2-PG) were investigated in 64 commercial Chinese Baijiu including soy sauce aroma-type Baijiu (SSB), strong aroma-type Baijiu (STB), and light aroma-type Baijiu (LTB), via chiral gas chromatography ( $\beta$-cyclodextrin). The natural enantiomeric distribution and concentration of 1,2-PG in various baijiu were studied to evaluate whether the distribution and content of the two isomers of 1,2-PG were correlated with the aroma type and storage year. The results showed that 1,2-PG has a high enantiomeric ratio and the (S)-configuration predominated in SSB. The average $S / R$ enantiomeric ratio of this compound in SSB was approximately 87:13 ( \pm 3.17$)$, with an average concentration of $52.77( \pm 23.70) \mathrm{mg} / \mathrm{L}$ for the $(S)$-configuration and $8.72( \pm 3.63) \mathrm{mg} / \mathrm{L}$ for the $(R)$-enantiomer. The $(R)$-configuration was predominant in the STB, whereas neither $(S)$ nor $(R)$-form of 1,2-PG were detected in LTB. The content of the two configurations of 1,2-PG in the JSHSJ vintage of SSB showed a wave variation, with an average $S / R$ enantiomeric ratio of 89:11 $( \pm 1.15)$. The concentration of $(R)-1,2-\mathrm{PG}$ in XJCTJ vintage liquors showed an upward and then downward trend with aging time, with an overall downward trend, and the concentration of (S)-form showed a wavy change with an overall upward trend. Except for the LZLJ-2019 vintage where both $(R)$ and (S)-1,2-PG were present, all other samples only existed $(R)$-form, and a decreasing trend of $(R)$-enantiomer with aging time was observed. The enantiomeric ratio of 1,2-PG might be one of the potential markers for adulteration control of Baijiu as industrial 1,2-PG usually presented in the racemic mixture. Sensory analysis revealed olfactory thresholds of $4.66 \mathrm{mg} / \mathrm{L}$ and $23.92 \mathrm{mg} / \mathrm{L}$ for the $(R)$ - and $(S)$-configurations in pure water respectively. GC-O showed both enantiomers exhibited different aromatic nuances.

Keywords: 1,2-Propylene glycol; enantiomer; chiral gas chromatography; Baijiu; aroma

\section{Introduction}

Chinese Baijiu is one of the six most famous distilled spirits in the world, with a history of more than 2000 years, and it plays an important role in Chinese traditional culture [1]. Baijiu is a clear and transparent fermented alcoholic beverage, usually with an ethanol content of $38-65 \%$ by volume and containing many trace flavor substances. The unique flavor components in Chinese Baijiu are produced from many resources, such as materials, fermentation, distillation, and the aging process, and which will determine its aroma style [2,3]. There are 12 aroma types of Baijiu and more than 2400 flavor compounds found in different Baijiu samples, some of which are chiral flavor substances $[4,5]$.

Chirality is one of the important properties of nature. Different isomers of chiral compounds may exhibit different properties, including aroma property and intensity [6-8]. For example, the four stereoisomers of 2-methyl-tetrahydrofuran-3-thiol acetate were found to present perceptible differences in aroma characteristics and intensities in our previous work [9]. The $R$-configuration of 3-mercapto-1-hexanol in wine has a grapefruit 
aroma with an olfactory threshold of $50 \mathrm{ng} / \mathrm{L}$, while the $S$ configuration has a passion fruit aroma with an olfactory threshold of $60 \mathrm{ng} / \mathrm{L}[10,11]$. In recent years, there has been an increasing demand and emphasis on identifying enantiomers in the food industry, especially for flavors, fragrances and alcoholic beverages [12]. The knowledge of the natural enantiomeric distribution and concentration of chiral compounds in alcoholic beverages can be a valuable way to assess food quality, authenticity, safety and evaluate processing and storage time effects, as well as confirm the geographical origin of product matrices, etc. [13-17]. Langen et al. studied the natural concentration and enantiomeric distribution of 1,2-PG in various wines to evaluate its advantages as a potential sign of wine aroma adulteration [18].

As far as we know, more than 40 chiral compounds have been investigated in alcoholic beverages, including five alcohols, such as 2,3-butanediol [19,20], 2-methyl-1butanol [21,22], 1-phenylethanol [23], 2-butanol [19], and 1,2-PG [18]. The 1,2-PG has an asymmetric chiral carbon atom with two different enantiomers (as in Figure 1). 1,2-PG is a colorless viscous liquid with good chemical stability and various applications. It is a polyol compound with certain hygroscopicity and is widely used as solvent, antifreeze, deicing agent, and humidifier in the food industry [24,25]. The addition of 1,2-PG to foods can retain a certain amount of moisture, meanwhile it does not increase the water activity, so it is often used as a lubricant to improve the sensory qualities of food [26]. 1,2-PG was found with a high level of $(R)$-form, in beer [27], wine [18,28], sparkling wine, at the concentration level of $\mathrm{mg} / \mathrm{L}$. 1,2-PG has been applied as a potential signature compound for authenticity verification in alcoholic beverages such as wine and beer [18,27]. Understanding the natural enantiomeric composition of 1,2-PG is important for the authenticity assessing in wine, so as in Baijiu. 1,2-PG was detected in raw liquor of Maotai flavour Baijiu [29] as well as the mechanized brewing of strong-flavor Baijiu [30].

(a)

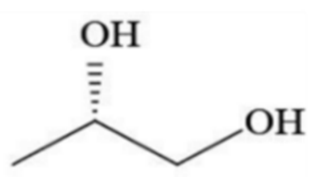

(b)

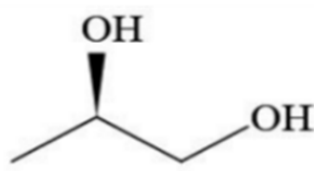

Figure 1. (a) (S)-1,2-PG (CAS No. 4254-15-3); (b) (R)-1,2-PG (CAS No. 4254-14-2).

The distribution and concentration of the two isomers of 1,2-PG in Baijiu are not clear. Therefore, the aim of this work was to isolate and determine (S)-1,2-PG and (R)-1,2-PG in SSB, STB, LTB and the corresponding vintages to explore whether the distribution and content of the two isomers of 1,2-PG are correlated with the aroma type and aging year.

\section{Materials and Methods}

\subsection{Chemicals}

Anhydrous ethanol (chromatographic grade, 99.97\%), Tianjin kemiou Chemical Reagent Co., Ltd., Tianjin, China. anhydrous sodium sulfate (99\%) (Chengdu Jinshan Chemical Reagent Co., Chengdu, China). microfiltered water, SZ-93A Pure Water Distiller, (Shanghai Yarong Co, Shanghai, China). The chiral standards are as follows: (R)-1,2-PG, 98\%, (Shanghai Maclean Technology Co, Shanghai, China). (S)-1,2-PG, 99\%, ( \pm )-1,2-PG (racemic mixture, 50:50), 99\%, (TCI Shanghai Chemical Industry Development Co, Shanghai, China); 2-octanol, 98\%, Dr. Ehrensorfer, Germany. The standards were stored at $4{ }^{\circ} \mathrm{C}$ in a refrigerator.

\subsection{Samples}

The determination of (S)- and (R)-1,2-PG were carried out in 38 types of SSB, 16 of STB and 10 LTB. In this paper, the 64 samples were divided into two categories, in which SSB included commercial Baijiu products, JSHSJ vintage Baijiu (five-41 years), XJCTJ vintage Baijiu (one-11 years); STB included commercially available Baijiu products and LZLJ vintage Baijiu (two-nine years); and LTB included commercially available Baijiu products. 


\subsection{Sample Preparation}

A $5 \mathrm{~mL}$ of the experimental Baijiu sample was added directly into a centrifuge tube, and $1.86 \mathrm{~g}$ of anhydrous sodium sulfate was added, overnight, and then filtered through a $0.22 \mu \mathrm{m}$ organic system filter membrane. $10 \mu \mathrm{L}$ of the internal standard solution (2-octanol, $5 \mathrm{mg} / \mathrm{L}$ ) was added to $990 \mu \mathrm{L}$ of the Baijiu sample, filtered through a $0.22 \mu \mathrm{m}$ organic filter membrane into a $1.5 \mathrm{~mL}$ injection vial, and awaited GC analysis.

\subsection{Qualitation and Quantification of 1,2-PG Enantiomers}

The two enantiomers of 1,2-PG were determined using a Thermo Fisher gas chromatograph (TRACE 1300), and the treated Baijiu sample was injected into the injection port in a split mode (injection port temperature $250^{\circ} \mathrm{C}$, split ratio: 20:1). The column was CYCLOSIL-B ( $30 \mathrm{~m} \times 0.25 \mathrm{~mm} \times 0.25 \mu \mathrm{m}$, (Agilent Technologies Ltd., Santa Clara, CA, USA). The oven temperature was programmed at $50{ }^{\circ} \mathrm{C}$ for $1 \mathrm{~min}$, then increased at a rate of $3{ }^{\circ} \mathrm{C} / \mathrm{min}$ to $80^{\circ} \mathrm{C}$, and finally raised by $8{ }^{\circ} \mathrm{C} / \mathrm{min}$ to a final isotherm at $170{ }^{\circ} \mathrm{C}$, and maintained for $5 \mathrm{~min}$. Qualitative analysis: Determination of the configuration of $(S)$ - and (R)-1,2-PG in Baijiu: first, we compared the retention times with the standards of the two enantiomers to determine the elution sequences. Next, we added the (S)- and (R)-1,2-PG standards to the Baijiu samples, respectively, and observed the changes of the corresponding peak areas of the two conformations to determine the two peaks of the two conformations in the Baijiu samples. The quantification was performed using the calibration curve established in Baijiu. All experiments were repeated three times.

\subsection{GC-O Analysis and Evaluation}

The reference compounds were sniffed by GC-O by a team of trained researchers. The olfactory analysis was performed using a Thermo Fisher gas chromatograph (TRACE 1300), coupled with a sniffer (Brechbuhler AG, Switzerland) (SNIFFER 9100) equipped with a FID and a sniffing port connected to the column outlet via a splitter. GC effluent was combined with humidified $\mathrm{N}_{2}$ at the bottom of the glass-sniffing nose to avoid nasal dehydration. Each enantiomer standard of 1,2-PG was injected with $1 \mu \mathrm{L}$, injection temperature $\left(250^{\circ} \mathrm{C}\right.$ in split mode, split ratio: 20:1). The column was a CYCLOSIL-B $(30 \mathrm{~m} \times 0.25 \mathrm{~mm} \times 0.25 \mu \mathrm{m}$, Agilent Technologies Ltd., Santa Clara, CA, USA). The oven temperature was programmed at $50{ }^{\circ} \mathrm{C}$ for $1 \mathrm{~min}$, then increased at a rate of $3{ }^{\circ} \mathrm{C} / \mathrm{min}$ to $80{ }^{\circ} \mathrm{C}$, and finally raised by $8{ }^{\circ} \mathrm{C} / \mathrm{min}$ to a final isotherm at $170{ }^{\circ} \mathrm{C}$, and maintained for $5 \mathrm{~min}$. The carrier gas was high purity nitrogen with a column head pressure of $50 \mathrm{kpa}$.

\subsection{Aroma Thresholds}

Ten trained evaluators aged 22-29 (five males and five females) were selected for this experiment. Olfactory detection thresholds of $(R)$ - and (S)-1,2-PG were determined by sensory panel in microfiltered water, referring to the GB/T 22366-2008 sensory analysis methodology adopted (general guidelines for the determination of olfactory and flavor perception thresholds by the three-point option method (3-AFC)) and GB/T33406-2016 (Baijiu flavor GB/T33406-2016 (guidelines for determination of flavor thresholds of Baijiu) [31,32]. 3-AFC method: We rovided each panelist with six sets of samples, one reference sample and one known blank sample. Each set included two blanks and test compound solutions of known concentration. Each evaluation sample (including test compound solution of known concentration and blank sample) was marked with a random code of three to four digits. All the samples were poured into the clean tulip-shaped Baijiu glasses, then judged by the assessors at $20^{\circ} \mathrm{C} \pm 5{ }^{\circ} \mathrm{C}$. We compared the evaluation result of the evaluator with the actual type of the sample to determine whether the concentration of each flavor substance was answered correctly or not, and then calculated based on the statistical results. All experiments were carried out three times. The individual best estimate threshold (BET) of each assessor was calculated based on the geometric mean of the highest concentration sample that answered incorrectly and the adjacent higher-level concentration sample. $T_{B E T i}$ is the individual best estimate threshold; $A_{x}$ is the highest concentration sample that the 
evaluator answered incorrectly; $A_{x+1}$ is the Concentration of higher-level samples; $T_{B E T}$ is the Group threshold.

$$
\begin{gathered}
T_{B E T i}=\sqrt{A_{x} \times A_{x+1}} \\
T_{B E T}=\sqrt[n]{T_{B T E 1} \times T_{B E T 2} \ldots \times T_{B E T n}}
\end{gathered}
$$

\subsection{Statistical Analysis}

Data were organized according to the Microsoft Office Excel 2018 application, box line graphs, scatter plots, and histograms were produced by origin 2018 64Bit. An SPSS software (IBM SPSS Statistics 26.lnk) $t$-test was used to assess the significant differences in 1,2-PG levels in different aromatic Baijiu. The level of statistical significance was $5 \%$, $p<0.05$. The results of the sensory tests were statistically calculated according to the national standard specifications.

\section{Results and Discussion}

\subsection{Enantiomeric Distribution and Concentration of 1,2-PG}

The enantiomers of 1,2-PG were separated by chiral gas chromatography a with $\beta$-cyclodextrin phase. Figure 2 is the chromatogram of representative Baijiu samples of three different aromas: (a) is one of the Chromatogram of SSB; (b) is one of the chromatogram of STB; and (c) is one of the chromatogram of LTB. As shown in Figure 2, 1,2-PG enantiomers exhibited a good separation and (S)-1,2-PG was first eluted.

To further explore the enantiomers of 1,2-PG in Chinese Baijiu, qualitative and quantitative analysis was performed using GC-O. For both substances, good linearity was obtained in the ranges of $0.25-128 \mathrm{mg} / \mathrm{L}$ and $0.25-1024 \mathrm{mg} / \mathrm{L}$ for the $(R)$ - and $(S)$ configurations, respectively, with $\mathrm{R}^{2}$ values of 0.9996 and 0.9988 for the standard curves. Furthermore, the detection limits of the analytical methods were $0.791-1.471 \mathrm{mg} / \mathrm{L}$. The recoveries of $(R)-1,2-\mathrm{PG}$ chiral isomer standards ranged from $92.40 \%$ to $98.80 \%$ with the relative standard deviations (RSDs) of $0.81-1.54 \%$, and the recoveries of (S)-1,2-PG standards ranged from $98.40 \%$ to $100.85 \%$, with the RSDs of $1.09-5.06 \%$ (Table 1 ).

Table 1. Linear Ranges, Recovery Rates, $\mathrm{R}^{2}$, LOD, RSD.

\begin{tabular}{ccccccc}
\hline No. & Compounds & Linearity $(\mathbf{m g} / \mathrm{L})$ & $\mathbf{R}^{\mathbf{2}}$ & RSD (\%) & Recovery Rate (\%) & LOD (mg/L) \\
\hline 1 & $(R)-1,2-\mathrm{PG}$ & $0.25-128$ & 0.9995 & $0.81-1.54 \%$ & $92.40-98.80 \%$ & 1.471 \\
2 & $(S)-1,2-\mathrm{PG}$ & $0.25-1024$ & 0.9988 & $1.09-5.06 \%$ & $98.40-100.85 \%$ & 0.791 \\
\hline
\end{tabular}

Note: $\mathrm{R}^{2}$ : Correlation Coefficients, LOD: Limit of detection, RSD: relative standard deviation.

The enantiomeric distributions of 1,2-PG in 64 Baijiu of different aromas and vintages showed differences. The concentration levels of 1,2-PG ( $S$ and $R$ ) in SSB were higher than those of 1,2-PG in STB $(p<0.01)$. 1,2-PG enantiomers were not detected in LTB. In conclusion, the large variation in 1,2-PG content in different aromatic Baijiu suggested that the levels of 1,2-PG precursors may vary in different aromatic Baijiu. In addition, Langen et al. [18] quantified the 1,2-PG enantiomers in wines, where 1,2-PG has a high enantiomeric ratio and the $(R)$-configuration was dominant, with an $R / S$ ratio of about 90:10. Interestingly, the enantiomeric distribution of 1,2-PG in SSB is the opposite of that reported by Langen et al. in wine. The (S)-configuration was dominant in SSB, with an average ratio of $S / R$ of 87:13 $( \pm 3.17)$ in the SSB while in the vintage ones about 89:11 $( \pm 1.15)$ and 89:11 ( \pm 3.82 ), respectively (Table 2 ). The particular enantiomeric distribution of 1,2-PG with the great predominance of $(S)$-enatiomer in SSB could also be a potential marker for the adulteration.

The highest 1,2-PG content was found in SSB, with (S)-1,2-PG content from 22.33 $( \pm 1.75)$ to $101.88( \pm 2.72) \mathrm{mg} / \mathrm{L}$, while it was from 0 to $15.96( \pm 0.91) \mathrm{mg} / \mathrm{L}$ in STB; the $(R)-1,2-\mathrm{PG}$ content ranged between $2.74( \pm 0.06)$ and $19.18( \pm 2.75) \mathrm{mg} / \mathrm{L}$ in SSB, and 0 6.41 $( \pm 2.23) \mathrm{mg} / \mathrm{L}$ in STB (Table 3). The overall comparison of the two enantiomers of 1,2-PG in two aromatic types (SSB and STB) of Baijiu revealed significant differences between 
the enantiomers, with significantly higher concentrations of 1,2-PG in SSB than in STB $(p<0.01)$.
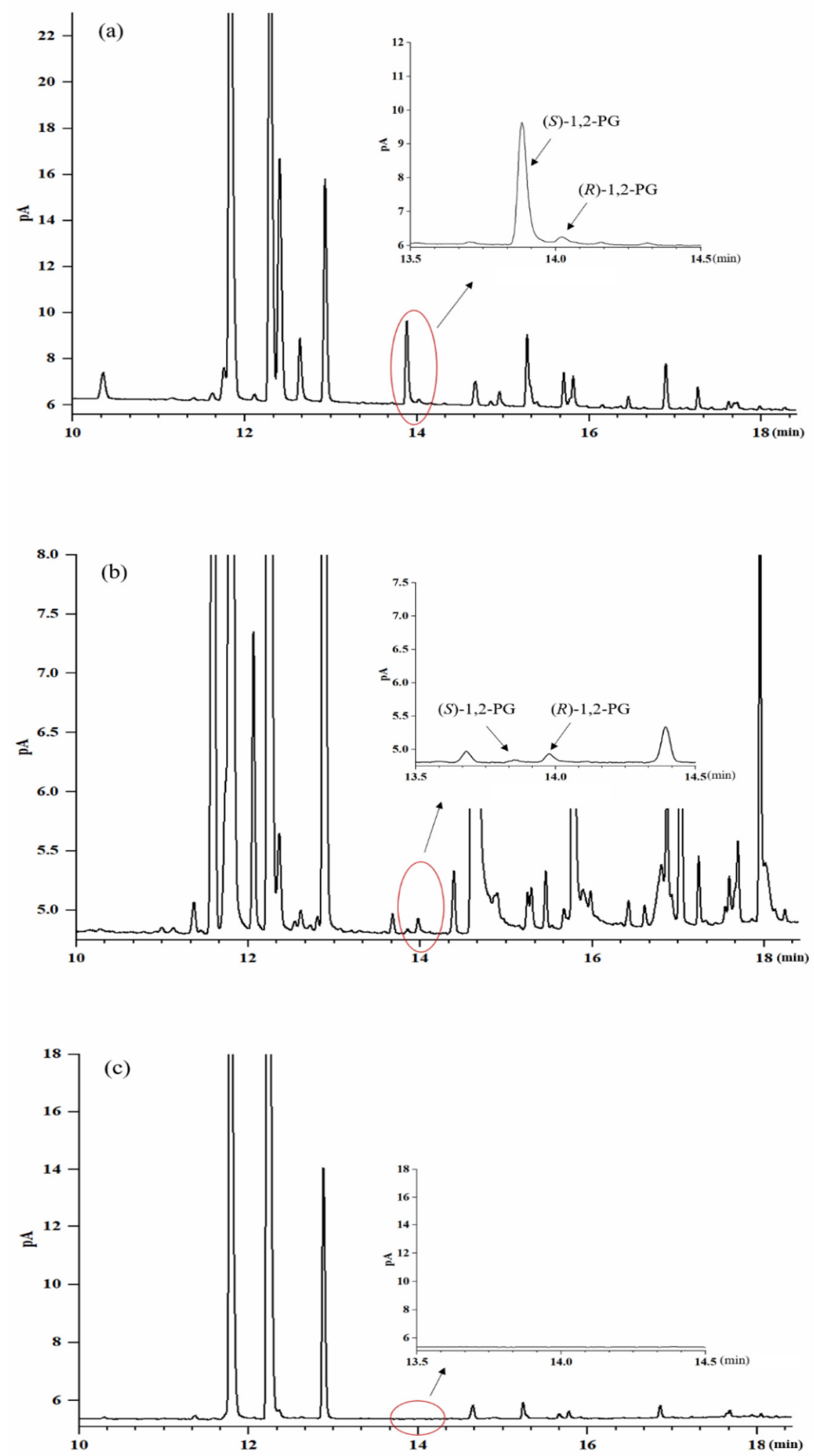

Figure 2. Separation chromatogram of 1,2-PG (1,2-Propylene Glycol) enantiomers in the representative Baijiu samples: (a) is one of SSB (soy sauce aroma-type Baijiu); (b) is one of STB (strong aroma-type Baijiu); (c) is one of LTB (light aroma-type Baijiu). 
Table 2. Average concentrations and ratios of 1,2-PG enantiomers in SSB.

\begin{tabular}{ccccc}
\hline & & \multicolumn{3}{c}{ Mean Concentration \pm Standard Deviation $(\mathrm{mg} / \mathrm{L})$} \\
\hline Category & Number of samples & $S$ & $R$ & $S / R$ \\
Baijiu products & 17 & $52.77 \pm 23.70^{\mathrm{a}}$ & $8.72 \pm 3.63^{\mathrm{a}}$ & $87: 13 \pm 3.17$ \\
$\begin{array}{c}\text { JSHJ Vintage Baijiu } \\
(1980-2016)\end{array}$ & 10 & $64.15 \pm 29.63^{\mathrm{a}}$ & $8.54 \pm 3.30^{\mathrm{a}}$ & $89: 11 \pm 1.15$ \\
$\begin{array}{c}\text { XJCTJ Vintage Baijiu } \\
(2010-2020)\end{array}$ & 11 & $63.01 \pm 24.56^{\mathrm{a}}$ & $10.08 \pm 3.53^{\mathrm{a}}$ & $89: 11 \pm 3.82$ \\
\hline
\end{tabular}

Note: *: Means in the same column with the same letter are not significantly different from each other $(p>0.05)$. SSB: soy sauce aroma-type Baijiu.

Table 3. Enantiomeric mean concentration * \pm standard deviation and ratio of 1,2-PG (1,2-Propylene Glycol) in different aromatic Baijiu.

\begin{tabular}{|c|c|c|c|c|}
\hline Sample & (S)-1,2-PG (mg/L) & $(R)-1,2-P G(m g / L)$ & ee & $S: R$ \\
\hline $\begin{array}{c}\text { SSB (soy sauce aroma-type Baijiu) } \\
\text { LM }\end{array}$ & $34.91 \pm 1.16^{\mathrm{d}}$ & $19.18 \pm 2.75^{a}$ & $29.09 \%$ & $65: 35$ \\
\hline JSHS & $90.71 \pm 2.73^{a}$ & $12.61 \pm 0.92^{\mathrm{cc}}$ & $75.59 \%$ & $88: 12$ \\
\hline JSJ & $29.41 \pm 3.95$ ee & $3.40 \pm 0.42^{\mathrm{cc}}$ & $79.28 \%$ & $90: 10$ \\
\hline ZJ & $73.15 \pm 11.05^{b b}$ & $11.40 \pm 2.36^{\mathrm{cc}}$ & $73.03 \%$ & $87: 13$ \\
\hline DYT2021 & $62.38 \pm 3.29^{\mathrm{cc}}$ & $8.66 \pm 0.35^{\mathrm{cc}}$ & $75.62 \%$ & $88: 12$ \\
\hline GT & $73.04 \pm 4.93^{b}$ & $8.55 \pm 0.53^{\mathrm{cc}}$ & $79.04 \%$ & $90: 10$ \\
\hline XJYZ & $24.57 \pm 1.65$ ee & $8.24 \pm 1.15^{\mathrm{cc}}$ & $49.78 \%$ & $75: 25$ \\
\hline QHL & $49.57 \pm 6.30^{\mathrm{dd}}$ & $7.30 \pm 0.61^{\mathrm{cc}}$ & $74.32 \%$ & $87: 13$ \\
\hline LJ & $91.81 \pm 10.34^{\text {аa }}$ & $7.54 \pm 0.42^{\mathrm{cc}}$ & $84.82 \%$ & $92: 8$ \\
\hline XJ1988 & $38.61 \pm 0.35^{\mathrm{dd}}$ & $6.89 \pm 0.36^{\mathrm{cc}}$ & $69.71 \%$ & $85: 15$ \\
\hline TCSP & $22.33 \pm 1.75^{\mathrm{e}}$ & $9.74 \pm 1.62^{\mathrm{cc}}$ & $39.25 \%$ & $70: 30$ \\
\hline GZJS & $39.44 \pm 2.44 \mathrm{dd}$ & $5.85 \pm 0.84^{\mathrm{cc}}$ & $74.15 \%$ & $87: 13$ \\
\hline MTWZ & $45.32 \pm 1.81 \mathrm{dd}$ & $8.53 \pm 0.38^{\mathrm{cc}}$ & $68.32 \%$ & $84: 16$ \\
\hline DYT & $89.54 \pm 0.48^{\text {aа }}$ & $7.47 \pm 0.05^{\mathrm{cc}}$ & $84.59 \%$ & $92: 8$ \\
\hline QJ1H & $43.60 \pm 1.16^{\mathrm{dd}}$ & $5.40 \pm 0.84^{\mathrm{cc}}$ & $77.96 \%$ & $89: 11$ \\
\hline MTCX & $28.64 \pm 0.92$ ee & $12.07 \pm 0.57^{\mathrm{cc}}$ & $40.72 \%$ & $70: 30$ \\
\hline MT43 & $60.02 \pm 1.04^{\mathrm{cc}}$ & $5.47 \pm 0.50^{\mathrm{cc}}$ & $83.29 \%$ & $92: 8$ \\
\hline JSHSJ-1980 & - & $3.26 \pm 0.14^{\mathrm{cc}}$ & - & - \\
\hline JSHS-1985 & $23.60 \pm 0.16^{\mathrm{ee}}$ & $2.74 \pm 0.06^{c}$ & $79.21 \%$ & $90: 10$ \\
\hline JSHSJ-1996 & $26.17 \pm 0.60$ ee & - & - & - \\
\hline JSHSJ-2002 & $66.04 \pm 3.12^{\mathrm{cc}}$ & $9.31 \pm 2.01^{\mathrm{cc}}$ & $75.30 \%$ & $88: 12$ \\
\hline JSHSJ-2004 & $41.15 \pm 2.09 \mathrm{dd}$ & $4.51 \pm 0.24^{\mathrm{cc}}$ & $80.25 \%$ & $90: 10$ \\
\hline JSHSJ-2010 & $99.24 \pm 4.73^{\text {аa }}$ & $11.40 \pm 1.29^{\mathrm{cc}}$ & $79.39 \%$ & $90: 10$ \\
\hline JSHSJ-2013 & $97.70 \pm 1.82$ & $9.54 \pm 2.20^{\mathrm{cc}}$ & $82.20 \%$ & $91: 9$ \\
\hline JSHSJ-2014 & $96.37 \pm 4.27$ aа & $12.49 \pm 0.89^{\mathrm{cc}}$ & $77.05 \%$ & $89: 11$ \\
\hline JSHSJ-2015 & $61.72 \pm 6.43^{\mathrm{cc}}$ & $9.28 \pm 1.48^{\mathrm{cc}}$ & $73.87 \%$ & $87: 13$ \\
\hline JSHSJ-2016 & $65.38 \pm 1.28^{\mathrm{cc}}$ & $9.08 \pm 2.06^{\mathrm{cc}}$ & $75.60 \%$ & $88: 12$ \\
\hline XJCTJ-2010 & $62.53 \pm 0.69^{\mathrm{cc}}$ & $5.83 \pm 0.48^{\mathrm{cc}}$ & $82.96 \%$ & $91: 9$ \\
\hline XJCTJ-2011 & $76.27 \pm 1.86^{b b}$ & $7.93 \pm 0.30^{\mathrm{cc}}$ & $81.17 \%$ & $91: 9$ \\
\hline XJCTJ-2012 & $77.93 \pm 1.16^{b b}$ & $7.36 \pm 0.02^{\mathrm{cc}}$ & $82.74 \%$ & $91: 9$ \\
\hline XJCTJ-2013 & $36.01 \pm 0.28^{\mathrm{dd}}$ & $16.18 \pm 0.52^{b}$ & $37.99 \%$ & 69:31 \\
\hline XJCTJ-2014 & $85.83 \pm 2.63^{a}$ & $7.67 \pm 0.29^{\mathrm{cc}}$ & $83.60 \%$ & $92: 8$ \\
\hline XJCTJ-2015 & $55.07 \pm 2.14^{c}$ & $9.83 \pm 0.33^{\mathrm{cc}}$ & $69.71 \%$ & $85: 15$ \\
\hline XJCTJ-2016 & - & - & - & - \\
\hline XJCTJ-2017 & - & - & - & - \\
\hline XJCTJ-2018 & $43.50 \pm 2.79 \mathrm{dd}$ & $13.50 \pm 0.15^{\mathrm{cc}}$ & $52.64 \%$ & $76: 24$ \\
\hline XJCTJ-2019 & $28.05 \pm 0.48^{\text {ee }}$ & $13.69 \pm 0.66^{\mathrm{cc}}$ & $34.39 \%$ & $67: 33$ \\
\hline XJCTJ-2020 & $101.88 \pm 2.72^{\mathrm{a}}$ & $8.74 \pm 0.50^{\mathrm{cc}}$ & $84.20 \%$ & $92: 8$ \\
\hline
\end{tabular}


Table 3. Cont.

\begin{tabular}{|c|c|c|c|c|}
\hline Sample & (S)-1,2-PG (mg/L) & (R)-1,2-PG (mg/L) & ee & $S: R$ \\
\hline \multicolumn{5}{|c|}{ STB (strong aroma-type Baijiu) } \\
\hline LZLJ-TOUQ & $12.95 \pm 0.11^{\mathrm{a}}$ & $3.45 \pm 0.14^{\mathrm{a}}$ & $57.93 \%$ & $79: 21$ \\
\hline LZLJ-TEQ & - & $2.86 \pm 0.08^{\mathrm{a}}$ & - & - \\
\hline LZLJEQ & - & - & - & - \\
\hline MZDQ & - & - & - & - \\
\hline JNC & - & $6.41 \pm 2.23^{\mathrm{a}}$ & - & - \\
\hline WLY & $15.96 \pm 0.91^{\mathrm{a}}$ & $4.55 \pm 0.57^{\mathrm{a}}$ & $55.65 \%$ & $78: 22$ \\
\hline GJ1573 & - & $2.50 \pm 0.07^{\mathrm{a}}$ & - & - \\
\hline SJF & - & - & - & - \\
\hline LZLJ-2012 & - & - & - & - \\
\hline LZLJ-2013 & - & - & - & - \\
\hline LZLJ-2014 & - & $2.62 \pm 0.38^{\mathrm{a}}$ & - & - \\
\hline LZLJ-2015 & - & $3.79 \pm 0.34^{\mathrm{a}}$ & - & - \\
\hline LZLJ-2016 & - & $5.46 \pm 0.27^{\mathrm{a}}$ & - & - \\
\hline LZLJ-2017 & - & $5.27 \pm 0.47^{\mathrm{a}}$ & - & - \\
\hline LZLJ-2018 & - & $5.22 \pm 0.61^{\mathrm{a}}$ & - & - \\
\hline LZLJ-2019 & $13.09 \pm 0.22^{\mathrm{a}}$ & $5.90 \pm 0.18^{\mathrm{a}}$ & $37.85 \%$ & 69:31 \\
\hline \multicolumn{5}{|c|}{ LTB (light aroma-type Baijiu) } \\
\hline LBFJ & - & - & - & - \\
\hline FJQH20 & - & - & - & - \\
\hline FJQXMR & - & - & - & - \\
\hline FJBF & - & - & - & - \\
\hline JXB & - & - & - & - \\
\hline YTX1988 & - & - & - & - \\
\hline FPLJ & - & - & - & - \\
\hline HXEGT & - & - & - & - \\
\hline NLSCN & - & - & - & - \\
\hline NLSEGT & - & - & - & - \\
\hline
\end{tabular}

Note: -: Not detected in the sample; ${ }^{*}$ Means in the same column with the same letter are not significantly different from each other $(p>0.05)$, the different letters indicate that the differences between the groups are significant $(p<0.05)$. Different letters $(\mathrm{a}, \mathrm{b}, \mathrm{c}, \mathrm{d}, \mathrm{e})$ indicate the significance of the analysis of variance.

The production of SSB is based on the production of Daqu (ferments), followed by seven repeated fermentations [33,34]. The concentrations of $(S)$ - and $(R)-1,2-P G$ in SSB were significantly higher than those of in STB and LTB, which may be related to the Daqu production and fermentation temperature of SSB. The concentrations and enantiomeric ratios of $(S)$-and $(R)-1,2-P G$ in the SSB products were significantly different $(p<0.01)$ (Figure $3 a, b)$. The $(S)$-configuration was predominant, with an average concentration around $52.77 \mathrm{mg} / \mathrm{L}$ and an average enantiomeric percentage around $87 \%$. The $(R)$-configuration concentration was low, with an average concentration about $8.72 \mathrm{mg} / \mathrm{L}$ and an average enantiomeric percentage of about $13 \%$. The concentration of $(R)-1,2-\mathrm{PG}$ in JSHSJ vintage showed a wave change with aging time and an overall decreasing trend, while the concentration of (S)-1,2-PG showed an increasing and then decreasing trend with aging time and an overall decreasing trend. The concentration of (R)-1,2-PG in the XJCTJ vintage was increasing and then decreasing with time, with an overall decreasing trend, and the concentration of (S)-1,2-PG was a wavy variation with an overall increasing trend (Figure 3c,d).

Unlike SSB, STB is made with the Daqu at a medium temperature, followed by alcoholic fermentation at $32-35{ }^{\circ} \mathrm{C}$ [2]. The STB is mainly distributed in the Sichuan Basin (SCB)where is characterized by warmth and high humidity throughout the year [35]. Differences in the concentrations of $(S)$-and $(R)-1,2-\mathrm{PG}$ were found in the studied STB (Figure 4a) $(p>0.05)$. (R)-1,2-PG was dominant, with only (S)-1,2-PG in some of the Baijiu samples, such as LZLJTQ and WLY, and LZLJ-2019. 1,2-PG enantiomers were not detected in some samples. Moreover, among the STB vintage Baijiu, all samples had only $(R)-1,2-$ PG except for the LZLJ-2019 vintage, which had both (R)-and (S)-1,2-PG. Meanwhile, a decreasing trend of $(R)-1,2-\mathrm{PG}$ with aging time was observed in the STB vintage Baijiu (Figure $4 \mathrm{~b}$ ), probably due to the involvement of alcohols in the esterification reaction. 
(a)

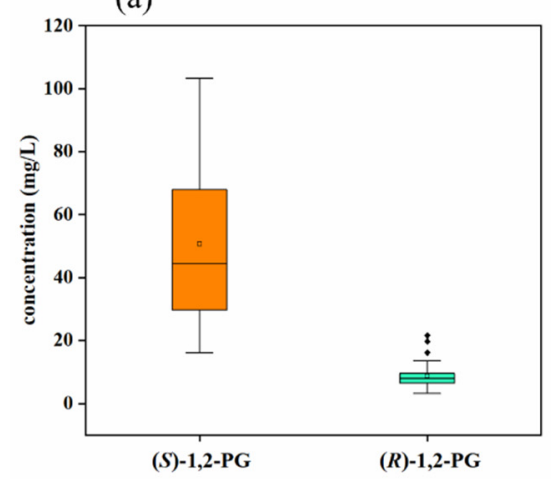

(c)

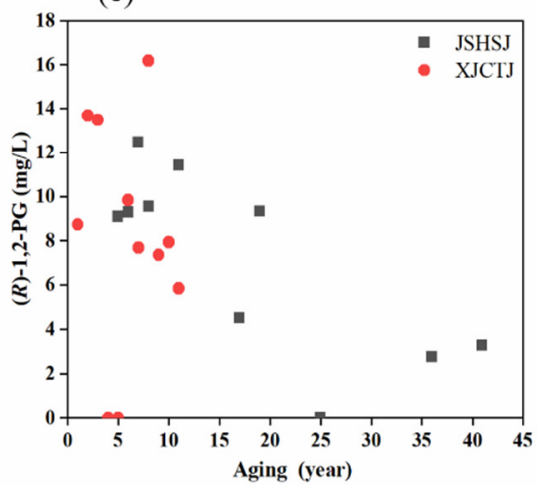

(b)

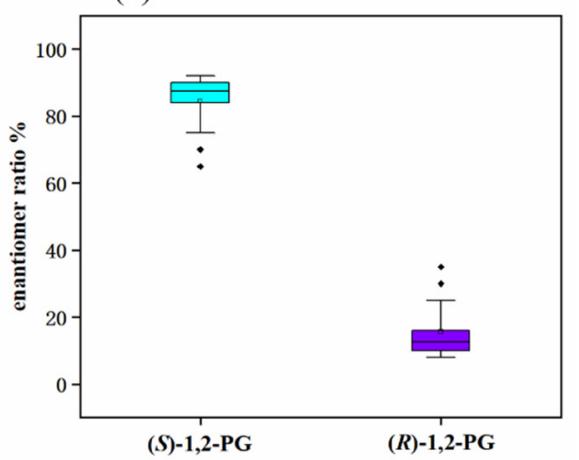

(d)

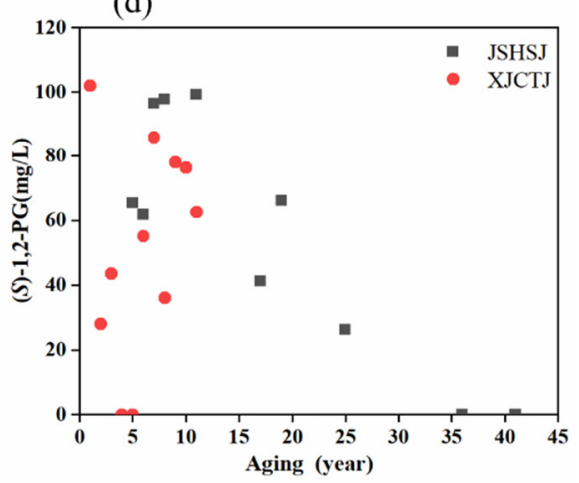

Figure 3. (a) Box line plot of 1,2-PG enantiomer content in SSB; (b) box line plot of 1,2-PG enantiomer ratio in SSB; (c) variation of (R)-1,2-PG content in SSB vintage Baijiu; (d) variation of (S)-1,2-PG content in SSB vintage Baijiu.

Compared to SSB and STB, LTB is produced mainly by low-temperature Daqu, and lower alcoholic fermentation temperatures [2]. LTB's representative brands, such as FJ, and HXEGT, have a pure and mild flavor with mellow sweetness and a fresh aftertaste [33]. Interestingly, none of the 1,2-PG enantiomers was detected in the studied LTB.

In order to better understand the variation of $(R)-1,2-\mathrm{PG}$ and (S)-1,2-PG content in the different aroma-types of Baijiu, the results of the study was processed as a heat map, and the color (from blue to red) indicated the variation of the relative intensity from low to high. In addition, a dendrogram of the relationship between the enantiomeric contents of 1,2-PG in different types of Baijiu was also plotted, as shown in (Figure 4c). Cluster analysis also showed a trend: 1,2-PG was somewhat differentiated among the different aromatic Baijiu. The results indicated that the two 1,2-PG enantiomers had some differences in different aromatic Baijiu.

These results highlighted the complexity of the sources of the two enantiomers, which might come from different biosynthetic and chemical pathways. The production of liquor is processed from traditional natural fermentation which involves hundreds of microbial communities, and there are certain differences in the microbial communities of different flavors of Baijiu [36]. 1,2-PG, a flavor substance in Baijiu, has been found to be metabolized by certain microorganisms. As a natural product, the accumulation of 1,2-PG was reported in the cultivation of Clostridium thermobutylicum [37]. Suzuki and Onishi et al. found that many different genera and species of yeast could convert L-rhamnose to 1,2-PG under aerobic conditions [38]. Schütz and Radler found that Lactobacillus shortum could also produce 1,2-PG [39]. However, the origin of 1,2-PG is not clear, each enantiomer has a different pathway, and the elucidation of their metabolic pathways requires specific research. The enantiomeric distinction allows for a more accurate evaluation of fragrance and aromatic components. Furthermore, each enantiomer of many chiral compounds is known to lead to different sensory responses in consumers $[20,23]$. 


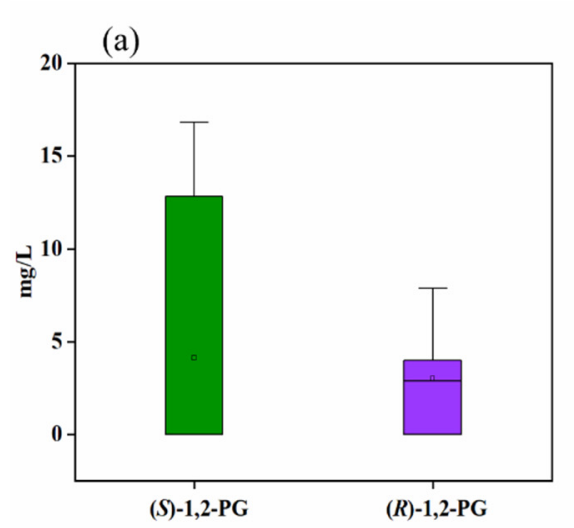

(b)

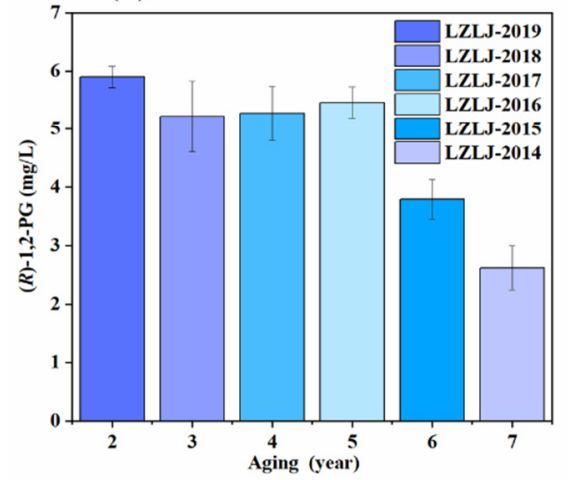

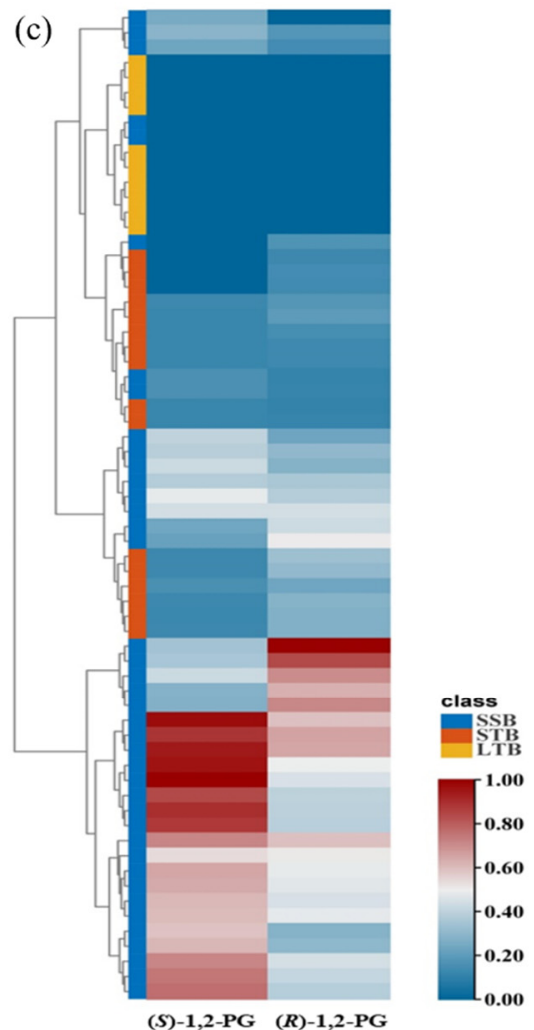

$(S)-1,2-\mathrm{PG} \quad(R)-1,2-\mathrm{PG}$

Figure 4. (a) Box line plot of 1,2-PG enantiomer content in STB; (b) histogram of vintage (R)-1,2-PG enantiomer content with aging year in STB; (c) thermogram analysis of 1,2-PG enantiomers in Baijiu.

\subsection{Odor Characteristics of 1,2-PG}

The 1,2-PG enantiomers standards used in this study were of high purity $(98 \%, 99 \%)$ and no odorous impurities were detected by the 10 judges who performed the analysis by GC-O. The (R)-1,2-PG was described as having a faint alcoholic, fruity, sweet aroma; while the $(S)$-configuration was characterized by an aromas of wood and alcohol.

The detection threshold of (S)-1,2-PG in pure water was determined by the sensory panel to be $23.92 \mathrm{mg} / \mathrm{L}$, about five times that of the $(R)$-form (Table 4$)$. These results showed that the stereochemistry of the molecules has a certain influence on their perception, which has been confirmed in several studies [21,40,41].

Table 4. Olfactory thresholds of 1,2-PG in water and its odor characteristics.

\begin{tabular}{ccc}
\hline Compounds & Odor Threshold (in Pure Water) $\mathbf{m g} / \mathbf{L}$ & Odor Characteristics \\
\hline$(S)-1,2-\mathrm{PG}$ & 23.92 & $\begin{array}{c}\text { Aromas of wood, } \\
\text { faint alcoholic } \\
\text { Sweet aroma, fruity, } \\
\text { faint alcoholic }\end{array}$ \\
\hline
\end{tabular}

\section{Conclusions}

The natural enantiomeric distribution and concentration of 1,2-PG enriched the current knowledge of 1,2-PG enantiomers in Baijiu. The content of 1,2-PG depended on the aroma style and age of the liquor to varying degrees, and its content is in the decreasing order of SSB, STB, and LTB. The concentration of the two configurations of 1,2-PG in the JSHSJ vintage of SSB showed a wave variation and an overall downward trend, with an average $S / R$ enantiomeric ratio of 89:11 $( \pm 1.15)$; the content of $(R)-1,2-\mathrm{PG}$ in the XJCTJ vintage Baijiu generally tended to decrease, while the concentration of $(S)-1,2-P G$ generally tended to increase with time, with an average $S / R$ enantiomer ratio in XJCTJ vintage Baijiu of 89:11 
( \pm 3.82$)$. In addition, the concentration of $(R)-1,2-\mathrm{PG}$ in STB vintage Baijiu decreased with time. In particular, the enantiomeric ratios showed a predominance of the $(S)$-configuration in SSB, while the $(R)$-configuration dominated in STB. Sensory analysis showed that the olfactory threshold of (S)-1,2-PG was about five times that of (R)-1,2-PG (4.66 mg/L). The two configurations have different odors. The enantiomeric ratio of 1,2-PG might be a potential marker for adulteration of Baijiu, as the industrial 1,2-PG is usually present in racemic form. Besides, it could also be a potential marker to differentiate the aroma styles of Baijiu based on its different enantiomeric ratios. Therefore, the enantiomeric ratio of 1,2-PG may be a new way to identify the quality and the aroma styles of Baijiu. The results of this study will further facilitate quality control of Chinese Baijiu.

\section{Patents}

There is a patent which we are preparing resulting from the work reported in this manuscript.

Author Contributions: Conceptualization and methodology, Y.D.; software, H.X.; validation, H.X.; formal analysis, Y.D. and H.X.; investigation and data curation, H.X.; writing-original draft preparation, H.X. and Y.D.; writing-review and editing, Y.D.; visualization, S.Q. and X.Z.; supervision, Y.D. and B.S.; project administration, Y.D. and S.Q.; funding acquisition, Y.D. All authors have read and agreed to the published version of the manuscript.

Funding: This research was funded by National Natural Science Foundation of China, grant number 31801509, 32060518; Guizhou Province Science and Technology Project, grant number [2019]1078, [2022]006, [2018]5781, ZK [2021]180; Open Project of Beijing Advanced Innovation Center for Food Nutrition and Human Health, grant number 20182010; Training Program of Guizhou University, grant number [2017]5788-22; Guizhou University Talent Introduction Research Project, grant number [2017]42.

Conflicts of Interest: The authors declare no conflict of interest. The funders had no role in the design of the study; in the collection, analyses, or interpretation of data; in the writing of the manuscript, or in the decision to publish the results.

\section{References}

1. Jia, W.; Fan, Z.B.; Du, A.; Li, Y.L.; Zhang, R.; Shi, Q.Y.; Shi, L.; Chu, X.G. Recent advances in Baijiu analysis by chromatography based technology-A review. Food Chem. 2020, 324, 126899. [CrossRef]

2. Liu, H.L.; Sun, B.G. Effect of Fermentation Processing on the Flavor of Baijiu. J. Agric. Food Chem. 2018, 66, 5425-5432. [CrossRef]

3. Han, S.H.; Zhang, W.W.; Li, P.Y.; Li, X.; Liu, J.X.; Xu, B.C.; Luo, D. Characterization of aromatic liquor by gas chromatography and principal component analysis. Anal. Lett. 2017, 50, 777-786. [CrossRef]

4. Xu, Y.Q.; Zhao, J.R.; Liu, X.; Zhang, C.S.; Zhao, Z.G.; Li, X.T.; Sun, B.G. Flavor mystery of Chinese traditional fermented Baijiu. Food Chem. 2021, 369, 130920. [CrossRef]

5. Xu, Y.Q.; Sun, B.G.; Fan, G.S.; Teng, C.; Xiong, K.; Zhu, Y.P.; Li, J.L.; Li, X.T. The brewing process and microbial diversity of strong flavour Chinese spirits: A review. J. Inst. Brew. 2017, 123, 5-12. [CrossRef]

6. D'Orazio, G.; Fanali, C.; Asensio-Ramos, M.; Fanali, S. Chiral separations in food analysis. TrAC Trends Anal. Chem. 2017, 96, 151-171. [CrossRef]

7. Engel, K. Chirality: An important phenomenon regarding biosynthesis, perception, and authenticity of flavor compounds. J. Agric. Food Chem. 2020, 68, 10265-10274. [CrossRef]

8. Alvarez-Rivera, G.; Bueno, M.; Ballesteros-Vivas, D.; Cifuentes, A. Chiral analysis in food science. TrAC Trends Anal. Chem. 2020, 123, 115761. [CrossRef]

9. Dai, Y.F.; Shao, J.Q.; Yang, S.X.; Sun, B.G.; Liu, Y.G.; Ning, T.; Tian, H.Y. Enantioselective Syntheses and Sensory Properties of 2-Methyl-tetrahydrofuran-3-thiol Acetates. J. Agric. Food Chem. 2015, 63, 464-468. [CrossRef]

10. Tominaga, T.; Niclass, Y.; Frérot, E.; Dubourdieu, D. Stereoisomeric distribution of 3-Mercaptohexan-1-ol and 3-Mercaptohexyl acetate in dry and sweet white wines made from Vitis vinifera (Var. Sauvignon blanc and semillon). J. Agric. Food Chem. 2006, 54, 7251-7255. [CrossRef]

11. Chen, L.; Capone, D.L.; Jeffery, D.W. Chiral analysis of 3-sulfanylhexan-1-ol and 3-sulfanylhexyl acetate in wine by highperformance liquid chromatography-tandem mass spectrometry. Anal. Chim. Acta 2017, 998, 83-92. [CrossRef]

12. Elbashir, A.A.; Aboul-Enein, H.Y. Multidimensional gas chromatography for chiral analysis. Crit. Rev. Anal. Chem. 2018, 48, 416-427. [CrossRef]

13. Ebeler, S.E. Enantiomeric analysis as a tool for authentication of foods and beverages. ACS Symp. Ser. 2006, 952, 39-49.

14. Tiritan, M.E.; Fernandes, C.; Maia, A.S.; Pinto, M.; Cass, Q.B. Enantiomeric ratios: Why so many notations? J. Chromatogr. A 2018, 1569, 1-7. [CrossRef] 
15. Marchelli, R.; Dossena, A.; Palla, G. The potential of enantioselective analysis as a quality control tool. Trends Food Sci. Technol. 1996, 7, 113-119. [CrossRef]

16. Rocco, A.; Aturki, Z.; Fanali, S. Chiral separations in food analysis. TrAC Trends Anal. Chem. 2013, 52, 206-225. [CrossRef]

17. Ribeiro, C.; Goncalves, R.; Tiritan, M.E. Separation of enantiomers using gas chromatography: Application in forensic toxicology, food and environmental analysis. Crit. Rev. Anal. Chem. 2020, 51, 787-811. [CrossRef]

18. Langen, J.; Fischer, U.; Cavalar, M.; Coetzee, C.; Wegmann-Herr, P.; Schmarr, H. Enantiodifferentiation of 1,2-propanediol in various wines as phenylboronate ester with multidimensional gas chromatography-mass spectrometry. Anal. Bioanal. Chem. 2016, 408, 2425-2439. [CrossRef]

19. Ebeler, S.E.; Sun, G.M.; Vickers, A.K.; Stremple, P. Gas chromatographic analysis of chiral aroma compounds in wine using modified cyclodextrin stationary phases and solid phase microextraction. ACS Symp. Ser. 2001, 794, 45-56.

20. Barba, C.; Flores, G.; Herraiz, M. Stereodifferentiation of some chiral aroma compounds in wine using solid phase microextraction and multidimensional gas chromatography. Food Chem. 2010, 123, 846-851. [CrossRef]

21. Cameleyre, M.; Lytra, G.; Tempere, S.; Barbe, J. 2-Methylbutyl acetate in wines: Enantiomeric distribution and sensory impact on red wine fruity aroma. Food Chem. 2017, 237, 364-371. [CrossRef] [PubMed]

22. Matheis, K.; Granvogl, M.; Schieberle, P. Quantitation and enantiomeric ratios of aroma compounds formed by an ehrlich degradation ofl-Isoleucine in fermented foods. J. Agric. Food Chem. 2016, 64, 646-652. [CrossRef] [PubMed]

23. Lasekan, O. Enantiomeric differentiation of three key volatile compounds in three different palm wines (Elaeis guineensis, Borassus flabellifer and Nypa fruticans). CYTA J. Food. 2018, 16, 70-75. [CrossRef]

24. Nonaka, D.; Fujiwara, R.; Hirata, Y.; Tanaka, T.; Kondo, A. Metabolic engineering of 1,2-propanediol production from cellobiose using beta-glucosidase-expressing E. Coli. Bioresour. Technol. 2021, 329, 124858. [CrossRef] [PubMed]

25. Zhang, S.; Huo, Z.B.; Ren, D.Z.; Luo, J.; Fu, J.; Li, L.; Jin, F.M. Catalytic conversion of ethyl lactate to 1,2-propanediol over CuO. Chin. J. Chem. Eng. 2016, 24, 126-131. [CrossRef]

26. Restrepo, J.B.; Bustillo, J.A.; Bula, A.J.; Paternina, C.D. Selection, Sizing, and Modeling of a Trickle Bed Reactor to Produce 1,2 Propanediol from Biodiesel Glycerol Residue. Processes 2021, 9, 479. [CrossRef]

27. Mochizuki, N.; Kikuchi, K.; Ikeda, M. Determination of enantiomers of 1,2-propanediol in beer by gas chromatography. Seibutsu Kogaku Kaishi 1997, 75, 339-342.

28. De Revel, G.; Martin, N.; Pripis-Nicolau, L.; Lonvaud-Funel, A.; Bertrand, A. Contribution to the knowledge of malolactic fermentation influence on wine aroma. J. Agric. Food Chem. 1999, 47, 4003-4008. [CrossRef]

29. Cai, X.; Shen, Y.; Chen, M.; Zhong, M.; Zhou, Y.; Luo, A. Characterisation of volatile compounds in Maotai flavour liquor during fermentation and distillation. J. Inst. Brew. 2019, 125, 453-463. [CrossRef]

30. Yu, F.; Zhou, J.; Ming, H.; Wang, X.; Du, X.J. The flavor components of strong-flavor Baijiu of mechanization and traditional brewing methods. Food Ferment. Ind. 2021, 1-8.

31. National Standard of the People's Republic of China. Guidelines for Determination of Liquor Flavor Substances Threshold; GB/T 33406-2016; China Standards Press: Beijing, China, 2016.

32. National Standard of the People's Republic of China. Sensory Analysis-Methodology-General Guidance for Measuring Odour, Flavour and Taste Detection Thresholds by a Three-Alternative Forced-Choice (3-AFC) Procedure; GB/T 22366-2008; China Standards Press: Beijing, China, 2008.

33. Zheng, X.W.; Han, B.Z. Baijiu.Chinese liquor: History, classification and manufacture. J. Ethn. Foods. 2016, 3, 19-25. [CrossRef]

34. Jin, G.Y.; Zhu, Y.; Xu, Y. Mystery behind Chinese liquor fermentation. Trends Food Sci. Technol. 2017, 63, 18-28. [CrossRef]

35. Zhao, X.L.; Tang, Y.; Zhang, C.S. The geographical patterns of Chinese liquors during 1995-2004. J. Maps 2017, 13, 107-116. [CrossRef]

36. Wang, L.L.; Fan, S.S.; Yan, Y.; Yang, L.; Chen, S.; Xu, Y. Characterization of potent odorants causing a pickle-like Off-Odor in Moutai-Aroma type Baijiu by comparative aroma extract dilution analysis, quantitative measurements, aroma addition, and omission studies. J. Agric. Food Chem. 2020, 68, 1666-1677. [CrossRef]

37. Niu, W.; Kramer, L.; Mueller, J.; Liu, K.; Guo, J.T. Metabolic engineering of Escherichia coli for the de novo stereospecific biosynthesis of 1,2-propanediol through lactic acid. Metab. Eng. Commun. 2019, 8, e82. [CrossRef]

38. Suzuki, T.; Onishi, H. Aerobic Dissimilation of 1-Rhamnose and the Production of 1-Rhamnonic Acid and 1,2-Propanediol by Yeasts. Agric. Biol. Chem. 1968, 32, 888-893.

39. Schutz, H.; Radler, F. Anaerobic Reduction of Glycerol to Propanediol-1.3 by Lactobacillus brevis and Lactobacillus buchneri. System. Appl. Microbiol. 1984, 5, 169-178.

40. Pons, A.; Lavigne, V.; Landais, Y.; Darriet, P.; Dubourdieu, D. Distribution and organoleptic impact of sotolon enantiomers in dry white wines. J. Agric. Food Chem. 2008, 56, 1606-1610. [CrossRef]

41. Cretin, B.N.; Sallembien, Q.; Sindt, L.; Daugey, N.; Buffeteau, T.; Waffo-Teguo, P.; Dubourdieu, D.; Marchal, A. How stereochemistry influences the taste of wine: Isolation, characterization and sensory evaluation of lyoniresinol stereoisomers. Anal. Chim. Acta 2015, 888, 191-198. [CrossRef] 